\title{
Hemichannel composition and electrical synaptic transmission: molecular diversity and its implications for electrical rectification
}

\author{
Nicolás Palacios-Prado ${ }^{1,2}$, Wolf Huetteroth ${ }^{2,3}$ and Alberto E. Pereda ${ }^{1,2 *}$ \\ ' Dominick P. Purpura Department of Neuroscience, Albert Einstein College of Medicine, Bronx, NY, USA \\ ${ }^{2}$ Marine Biological Laboratory, Woods Hole, Massachusetts, MA, USA \\ ${ }^{3}$ Department of Neurobiology, University of Konstanz, Konstanz, Germany
}

Edited by:

Juan Andrés Orellana, Pontificia

Universidad Católica de Chile, Chile

Reviewed by:

Juan C. Saez, Universidad Catolica de Chile, Chile

Christian Giaume, Collège de

France, France

${ }^{*}$ Correspondence:

Alberto E. Pereda, Dominick P.

Purpura Department of

Neuroscience, Albert Einstein

College of Medicine, 1300 Morris

Park Ave, Bronx, New York,

NY 10461, USA

e-mail:

alberto.pereda@einstein.yu.edu
Unapposed hemichannels (HCs) formed by hexamers of gap junction proteins are now known to be involved in various cellular processes under both physiological and pathological conditions. On the other hand, less is known regarding how differences in the molecular composition of HCs impact electrical synaptic transmission between neurons when they form intercellular heterotypic gap junctions (GJs). Here we review data indicating that molecular differences between apposed HCs at electrical synapses are generally associated with rectification of electrical transmission. Furthermore, this association has been observed at both innexin and connexin $(C x)$ based electrical synapses. We discuss the possible molecular mechanisms underlying electrical rectification, as well as the potential contribution of intracellular soluble factors to this phenomenon. We conclude that asymmetries in molecular composition and sensitivity to cellular factors of each contributing hemichannel can profoundly influence the transmission of electrical signals, endowing electrical synapses with more complex functional properties.

Keywords: gap junction, connexin, innexin, electrical synapse, asymmetry, rectification

\section{INTRODUCTION}

Channels formed by connexin $(\mathrm{Cx})$ or pannexin proteins (connexon and pannexon, respectively) were shown to impact cellular properties and underlie various pathological processes by serving as conduits for ions and various autocrine and paracrine signaling molecules (Contreras et al., 2002; Bennett et al., 2003; Scemes et al., 2007; Iglesias et al., 2009; Figure 1A). Some of these channels can assemble into intercellular structures. That is, docking of two connexons or "hemichannels" (HC) from two adjacent cells form intercellular channels that cluster into structures called "gap junctions" (GJs; Goodenough and Paul, 2009; Figures 1B,C), which mediate intercellular communication between neighboring cells in virtually all tissues of deuterostomes (Hervé et al., 2005; Abascal and Zardoya, 2013). Invertebrate GJ proteins, however, are part of an unrelated gene family called innexins (Inxs; Starich et al., 1996; Ganfornina et al., 1999). Three Inx-like genes were subsequently found in the genome of vertebrates, which were named pannexins (Panxs; Panchin et al., 2000; Bruzzone et al., 2003). Interestingly, while Panxs were shown to form intercellular channels when overexpressed in oocytes (Bruzzone et al., 2003), there is little evidence so far supporting that they form GJ channels under physiological conditions (Dahl and Locovei, 2006; Sahu et al., 2014). Since Inxs form GJ channels in invertebrates (Hervé et al., 2005; Phelan, 2005), it is speculated that Panxs might have evolved to function mainly as HCs in vertebrates (Dahl and Locovei, 2006). On the other hand, recent evidence suggests that Inxs can also function as HCs or "innexons" (Dahl and Muller, 2014). Due to the current uncertainty of Panx-based GJ channels, the distinction between Inx and Panx has remained in the literature to distinguish GJ forming proteins in protostomes and cnidarians (Inx) vs. HC forming proteins in deuterostomes (Panxs) (Abascal and Zardoya, 2013).

Currently, we know that the family of $\mathrm{Cx}$ proteins in humans is composed by 21 genes (Söhl and Willecke, 2004) whereas Inxs represent 25 genes in C. elegans or eight genes in D. melanogaster (Adams et al., 2000; Altun et al., 2009). Gap junction channels formed by different Cxs and Inxs were shown to exhibit differential permeability and distinct electrophysiological properties providing diversity to gap junctional communication (Goodenough and Paul, 2009; Samuels et al., 2010). Notably, GJ channels can be either formed by the docking of identical (homotypic configuration; Figure 1B) or different (heterotypic configuration) HCs (Figure 1C), further enhancing the functional diversity of GJs. That is, at heterotypic channels, the molecular and functional singularities of each of the apposed/contributing HC (aHC) influence the properties of the intercellular channel and, furthermore, can potentially endow heterotypic channels with properties which could not be predicted from those displayed in homotypic configuration (Verselis et al., 1994; Oh et al., 1999). In other words, asymmetries in molecular composition of each aHC could profoundly influence intercellular communication, 
A
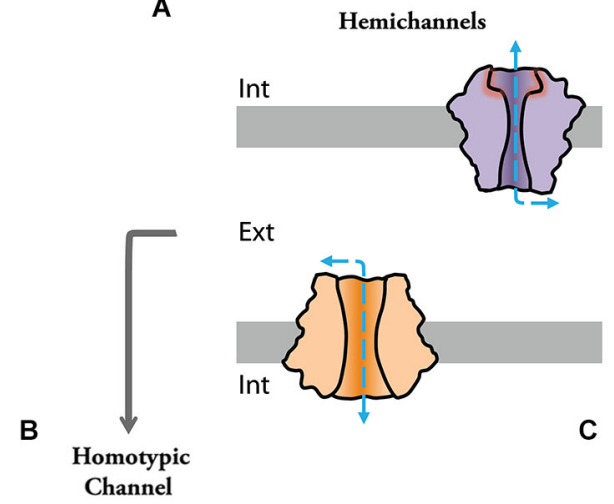

Ext

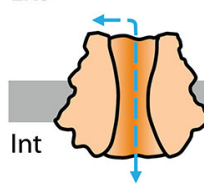

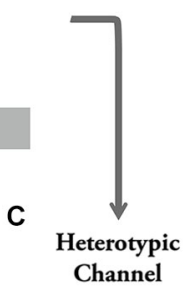

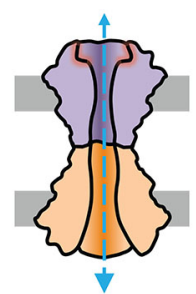

FIGURE 1 | Channels formed by connexin and innexin proteins can play functional roles as single "hemichannels" (HCs) or assemble into intercellular channels at gap junctions to provide cell-cell

communication. (A) Hemichannels can be molecularly different (note difference in color and shape) and act as conduits for the release of messenger molecules. (B) Identical HCs can assemble into intercellular "homotypic" gap junction channels. (C) Hemichannels of different molecular composition can assemble into intercellular "heterotypic" gap junction channels.

providing GJs with complex functional properties. In addition, many types of cells co-express several $\mathrm{Cx}$ or Inx isoforms with the potential to form heteromeric HCs. In the same scenario, different homomeric HCs can cluster into the same junctional plaque forming bi-homotypic GJs ( $\mathrm{Li}$ et al., 2008), in which HCs are docked with the same kind of HC, i.e., channels are homomeric homotypic. Heterotypic/heteromeric GJ channels are likely to be present in the retina, brain and peripheral system (Söhl et al., 2000; Vaney and Weiler, 2000). Twenty-one different $\mathrm{Cx}$ isoforms can potentially form 210 different heterotypic GJs, however not all different $\mathrm{Cx}$ isoforms are compatible with each other. More than forty functional heterotypic pairs have been found and analyzed so far (Palacios-Prado and Bukauskas, 2012). Although the expression of Drosophila Inxs was shown to overlap in some tissues (Stebbings et al., 2000) their functional compatibility remains, in contrast to Cxs, largely unexplored.

Gap junctions constitute the basis for electrical synaptic transmission in both vertebrate and invertebrate nervous systems (Bennett and Zukin, 2004; Pereda et al., 2013). Beyond their ability to allow the passage of small messenger molecules, neuronal GJs (or "electrical synapses") serve as low resistance pathways for the spread of electrical currents between coupled neurons, a key property for a cellular type that critically relies on electrical signaling (Bennett and Zukin, 2004; Connors and Long, 2004; Pereda et al., 2013). This article reviews data indicating

that molecular differences between aHC at electrical synapses are generally associated with rectification of electrical transmission (differential resistance to current flow in one vs. the other direction between two coupled cells), a voltage-dependent property of GJ channels that has been observed at both $\mathrm{Cx}$ and Inx-based electrical synapses (Furshpan and Potter, 1959; Auerbach and Bennett, 1969; Edwards et al., 1998; Rela and Szczupak, 2007; Rash et al., 2013). Here we discuss some molecular mechanisms underlying rectification of electrical transmission. We conclude that asymmetries in the molecular composition of individual HCs forming electrical synapses can strongly influence transmission of electrical signals between neurons coupled by GJs.

\section{BI-DIRECTIONALITY AND SYMMETRY OF ELECTRICAL TRANSMISSION}

Neurons operate by computing variations of the membrane potential evoked by synaptic currents and active processes, which are usually translated into trains of action potentials. The change in the membrane potential observed by the spread of presynaptic currents through GJs to a postsynaptic neuron is usually referred to as a "coupling potential". The amplitude of this "coupling potential" does not solely depend on the conductance of GJ channels but also on the passive properties determined by the capacitance and the input resistance $(\mathrm{R})$, which is directly proportional to the membrane resistance $\left(\mathrm{R}_{\mathrm{m}}\right)$, and indirectly proportional to the area of the membrane (size and geometry) of the coupled neurons (Figure 2A). The strength of an electrical synapse is generally expressed as the "coupling coefficient", a ratio that expresses the amplitude of the coupling potential normalized to the amplitude of the signal that originated it in a neighboring coupled cell (see equation in Figure 2A; this value is obtained once the capacitance of the membrane is charged, which is generally referred to as "steady state"). Electrical synaptic transmission is bidirectional and symmetric when the Rs of coupled cells are similar (Figure 2B). Gap junction channels at electrical synapses were shown in some cases to behave as electrical rectifiers, that is, to offer differential resistance to the flow of currents in one vs. the other direction across the junction between two coupled neurons. As a matter of fact, the first characterization of an unequivocally electrically mediated synapse came together with the description of electrical rectification (Furshpan and Potter, 1957, 1959). Rather than a simple bidirectional spread of electrotonic potential, the crayfish giant motor synapse transmitted depolarization signals from the giant axon to the motor fiber, but not in the opposite direction. Similarly, hyperpolarization signals were transmitted only from the motor fiber to the giant axon. Although the transmission of relative positive or negative potentials is unidirectional (only in one direction), rectifying junctions allow the spread of signals in either direction. In addition, postsynaptic signals reproduced the time course of presynaptic signals, and transmission was, surprisingly, voltage-dependent, thus challenging all the criteria established for chemical transmission. The rectification properties discovered in this preparation also helped to exclude the prevailing hypothesis of gross protoplasmic connections suggested earlier to explain symmetric electrotonic spread of current between 


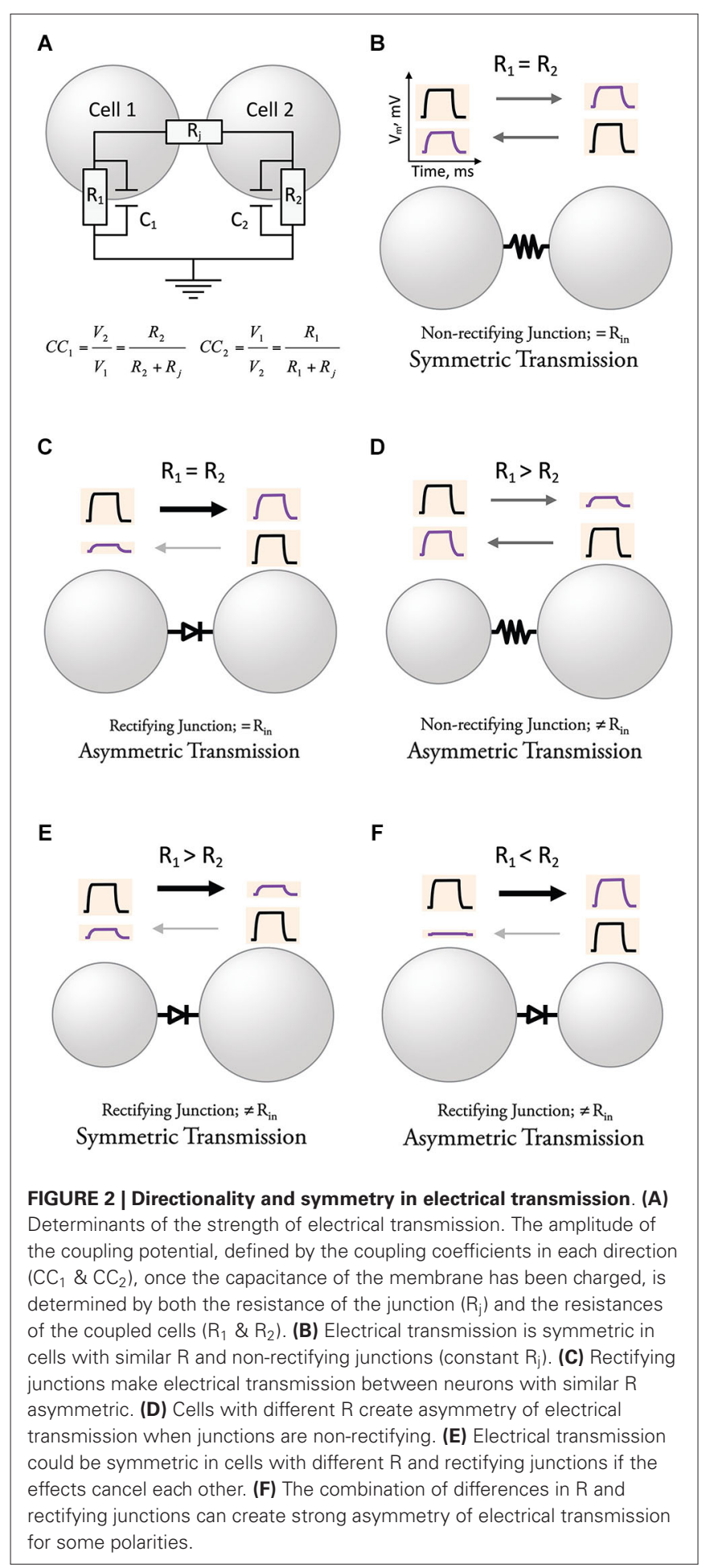

cardiac Purkinje cells (Weidmann, 1952) or among neurons of the lobster cardiac ganglion (Watanabe, 1958). Rectification was subsequently found in several electrical synapses in in vivo preparations exhibiting direction asymmetry of signal transfer (Smith et al., 1965; Auerbach and Bennett, 1969; Baylor and Nicholls, 1969; Ringham, 1975; Muller and Scott, 1981; Roberts et al., 1982; Margiotta and Walcott, 1983; Rash et al., 2013). Because of their properties, rectifying GJs can underlie asymmetric electrical transmission (Figure 2C). Asymmetry of electrical transmission does not necessarily require rectifying GJ channels, as differences in R of the coupled cells can make coupling coefficients stronger in the direction towards the cell with higher R (Figure 2D, Trenholm et al., 2013). Moreover, rectifying junctions can make a synapse bi-directional by counterbalancing the effect of differences in $\mathrm{R}$ of the coupled neurons (Figure 2E). Finally, the combination of rectifying junctions and differences in $\mathrm{R}$ of coupled cells can create strong asymmetric transmission (Figure 2F). Thus, although intimately related, directionality, rectification, and symmetry express different properties of electrical synaptic transmission and should not be considered interchangeable. In other words, electrical transmission could be: (1) bi-directional and asymmetric; (2) non-rectifying and markedly asymmetric; and (3) bidirectional and rectifying. Finally, while directionality and symmetry refer to electrical transmission (coupling potentials), the term rectification should be reserved to describe the asymmetric transjunctional current-voltage relationship of certain GJ channels.

\section{ASYMMETRY IN HEMICHANNEL COMPOSITION IS ASSOCIATED WITH RECTIFICATION OF ELECTRICAL TRANSMISSION}

The association of asymmetry of hemichannel composition and electrical rectification has been observed at both Inx- and Cxbased electrical synapses.

\section{INNEXIN-BASED ELECTRICAL SYNAPSES}

It was actually proposed that the formation of homomeric, homotypic channels is generally rare among fly Inxs (Phelan and Starich, 2001). Of the eight Inxs in Drosophila, a few were shown to form heterotypic GJs (reviewed in Hasegawa and Turnbull, 2014). Most information about electrical properties of heterotypic GJs in the fly brain exists for splice variants of the gene shakingB. Here we focus on two different circuits that both include shakB-based heterotypic electrical synapses in their architecture, the giant fiber system (GFS) and the antennal lobe. One forms a rather rigid reflex network, the other is a heavily modulated sensory information processor dealing with odors. In addition, we discuss the contribution of heterotypic GJs to memory formation in the fly brain and to C. elegans nervous system signaling.

The most complete picture of GJ function within a behavioral circuit is based on the GFS in Drosophila, an efficient escape reflex circuit that initiates a "jump and flight" response (reviewed in Allen et al., 2006). Threatening sudden stimuli can evoke a response in the giant fiber (GF) via giant commissural interneurons in the brain. The GF provides a fast connection of the brain with the ventral nerve cord in the thorax, where it forms mixed electrical and chemical synapses onto two cell types, a motor neuron that innervates a contralateral leg muscle, and an interneuron, which activates ipsilateral flight muscle motor neurons (Blagburn et al., 1999). The first evidence of the presence of GJs in this network stems from intracellular recordings on the GF with simultaneous brain stimulation and flight muscle recordings (Tanouye and Wyman, 1980). Subsequent work on 
the mutants shaking- $B^{2}\left(\operatorname{shak} B^{2}\right)$ and passover characterized the involved gene (Thomas and Wyman, 1984; Phelan et al., 1996; Sun and Wyman, 1996). It was later discovered that this shakB (or inx8) gene gives rise to five different splice variants, resulting in three confirmed protein isoforms: ShakB(Lethal), ShakB(Neural), and $\operatorname{ShakB}(\mathrm{Neural}+16)$, which has 16 more amino acids on the amino-terminus (Zhang et al., 1999). Interestingly, while ShakB (Lethal) is capable of functional homotypic channel formation in Xenopus oocytes, the ShakB(Neural) variant is not (Phelan et al., 1998). Ten years later the same lab used the GFS to provide first evidence that electrical rectification emerges from differential aHC composition and formation of heterotypic GJs (Phelan et al., 2008). They identified two ShakB variants being responsible for heterotypic GJs in the GFS: ShakB(Neural+16) in the presynaptic GF, and ShakB(Lethal) in the postsynaptic motor neuron and interneuron. A more recent study using oocytic expression of chimeric ShakB proteins suggested a role for the amino-terminal end of ShakB in voltage gating and electrical rectification (Marks and Skerrett, 2014).

Heterotypic GJs involving ShakB are also found in the antennal lobe. The antennal lobe is the first integration center of olfactory information in insects and shows structural similarity to the vertebrate olfactory bulb; within neuropilar substructures called glomeruli, the olfactory sensory neurons converge onto projection neurons (PNs), which in turn relay the olfactory information into higher brain regions (Wilson, 2013). The glomeruli are interconnected by a third class of antennal lobe neurons, the amacrine local interneurons (LNs). The majority are GABAergic and mainly provide lateral inhibition on the presynaptic terminal of the sensory neurons (iLNs), but some are excitatory (eLNs) and either cholinergic (Shang et al., 2007) or glutamatergic (Chou et al., 2010; Das et al., 2011). The cholinergic eLNs form chemical synapses onto iLNs and electrical synapses with PNs (Huang et al., 2010; Yaksi and Wilson, 2010). The mutant shakB ${ }^{2}$ abolishes electrical transmission between eLNs and PNs, and RT-PCR identified shakB transcripts in PNs (Yaksi and Wilson, 2010). $S_{\text {ShakB }}{ }^{2}$ (which affects both Neural variants) was successfully rescued by ectopic expression of ShakB(Neural) in adult flies. Since homomeric ShakB(Neural) HCs fail to form functional GJs (Phelan et al., 1998; Curtin et al., 2002), heterotypic interaction with another Inx, probably ShakB(Lethal) like in the GFS circuit, seems likely. A more targeted rescue in either PNs or eLNs will resolve on which side the $\mathrm{ShakB}$ (Neural) HC is essential; based on the electrical properties of the junction it would be expected on the eLN side.

There is also evidence for the presence of heterotypic GJs in the mushroom body (MB). The MB is regarded as the homologous structure of the vertebrate pallium in the brain of protostomes (Tomer et al., 2010) and is crucial for associative memory processes (Perisse et al., 2013). This paired neuropil consists mainly of about 2000 Kenyon cells on each side, which can roughly be subdivided in a dendritic calyx region, two orthogonal, elongated lobes, which contain the majority of presynaptic sites, and a peduncle that connects calyx with lobes. Prevailing sensory input to the Drosophila calyx is of olfactory nature, coming from the antennal lobe. Certain Kenyon cell subdivisions exhibit preferential roles in memory acquisition and in memory retrieval. The
$\mathrm{MB}$ is innervated by two large amacrine cells per hemisphere; the GABAergic anterior paired lateral cell (APL), which innervates all MB regions (Liu and Davis, 2009), and the serotonergic dorsal paired medial cell (DPM) which innervates peduncle and lobes only (Lee et al., 2011). A prominent role of the APL is to maintain signal sparseness by feedback inhibition (Lin et al., 2014), but it also shows involvement in labile appetitive memory (Pitman et al., 2011). The DPM is crucial for long-term memory consolidation, so its role can be separated from APL (Pitman et al., 2011). Both neurons seem to be electrically connected by heterotypic channels formed by Inx6 (DPM) and Inx7 (APL), especially in a subregion of the MB. This was inferred from contact marker expression and a combination of immunostainings, targeted RNAi expression against various Inxs in DPM and APL, dye coupling backfills and behavioral experiments (Wu et al., 2011; Pitman et al., 2011). Taken together, it is tempting to speculate that a rectifying electrical synapse between APL and DPM could contribute to generate a reverberant circuit, thus providing the ongoing cellular activity to consolidate a memory trace. The presence of this putative heterotypic channel is interesting for several reasons: it involves a novel pair of interacting Inxs, despite bigger spatial overlap between both contributing cells it seems to be segregated to a specific subcellular region, and, importantly, because of its potential contribution to a memory consolidation process.

The existence of numerous heterotypic GJs was suggested also to be the case for $C$. elegans Inxs, with the notable exception of UNC-7 and UNC-9, and possibly Inx14 with Inx8 or Inx9 (Simonsen et al., 2014). The unc-7 gene gives rise to three protein isoforms, and the homomeric heterotypic channel formed by the UNC-7S (or UNC-7b) isoform and UNC-9 was shown to be rectifying (Starich et al., 2009). Since both Inxs are widely expressed in nerve cells (Altun et al., 2009), and $\sim 10 \%$ of all synapses in C. elegans are electrical, this rectifying synapse might contribute significantly to direct signal transduction in the nematode nervous system. This is supported by the locomotion phenotype in mutants of both unc-7 and unc-9 (Starich et al., 1993; Barnes and Hekimi, 1997).

Gregarious behavior in C. elegans is determined by sensory integration in a hub-and-spoke circuit where the RMG neuron forms electrical synapses with many sensory neurons (Macosko et al., 2009). Activation or inhibition of this sensory integration induces gathering or solitary behaviors, respectively. Interestingly, RMG neurons are only labeled with the unc-7a promoter fragment while its sensory partners express different Inxs: IL2, ADL and AWB express UNC-9; IL2, ADL and ASK express Inx-18; and IL2, ADL and ASH express Inx19 (Altun et al., 2009). Since UNC-7S and UNC-9 are known to form rectifying heterotypic junctions (Starich et al., 2009), it is possible that electrical rectification is involved in sensory integration in the RMG hub-and-spoke circuit, and therefore heterotypic GJ might be involved in gregarious behavior of $C$. elegans. This type of circuit motif-one integrating hub neuron connected to many sensory neurons by electrical synapses-are present in large numbers (more than 15 different hubs) in the nematode nervous system and may be a conserved functional unit for coincidence detection (Rabinowitch et al., 2013). Finally, 
although the relationship with heterotypic Inx-based GJs still needs to be established, multiple rectifying electrical synapses have been described in various invertebrates, such as crayfish (Furshpan and Potter, 1959), horseshoe crab (Smith et al., 1965) and leech (Baylor and Nicholls, 1969; Muller and Scott, 1981).

\section{CONNEXIN-BASED ELECTRICAL SYNAPSES}

Although the presence of asymmetric transmission has been reported at electrical synapses between several vertebrate cell types, such as the inferior olive (Devor and Yarom, 2002), striatum (Venance et al., 2004), cochlear nucleus (Apostolides and Trussell, 2013) and thalamus (Haas et al., 2011) and reportedly involving in some of these cases asymmetry of GJ conductance (Devor and Yarom, 2002; Venance et al., 2004; Haas et al., 2011), electrical rectification was demonstrated in only a few cases (Auerbach and Bennett, 1969; Ringham, 1975; Rash et al., 2013). Recent evidence suggests that, as observed in invertebrates, electrical rectification is also associated with asymmetry in the molecular composition of aHCs. That is, electrical synapses at auditory afferents and the teleost Mauthner cell known as "Club endings" are formed by two homologs of mammalian Cx36 (considered the main synaptic $\mathrm{Cx}$ in mammals due to its widespread expression in neurons (Condorelli et al., 2000)), Cx35 and Cx34.7 (Rash et al., 2013). As a result of additional genome duplication (Volff, 2005), teleost fish have more than one homologous gene for most mammalian Cxs (Eastman et al., 2006). Remarkably, while Cx35 is restricted to presynaptic GJ hemiplaques (the portion of the GJ plaque contributed by each cell), Cx34.7 is restricted to postsynaptic hemiplaques, forming heterotypic junctions (Rash et al., 2013). In contrast to many different Cxs that are compatible to form heterotypic GJs, Cx36 is known so far to form only "homotypic" GJs (Teubner et al., 2000; Li et al., 2004). From an evolutionary point of view, the existence of compatible Cx36 teleost homologs that form heterotypic channels provided neurons with the ability to connect through GJs with more complex properties. Estimates of junctional conductance $\left(g_{\mathrm{j}}\right)$ between Club endings and the Mauthner cell revealed a four-fold difference between the antidromic (from the postsynaptic Mauthner cell to the presynaptic Club ending) and orthodromic (from the Club ending to the Mauthner cell) directions (Rash et al., 2013). This rectifying property is thought to play an important functional role by promoting cooperativity between different auditory afferents (see below).

\section{MECHANISMS UNDERLYING RECTIFICATION OF ELECTRICAL TRANSMISSION GAP JUNCTIONS AS DIODES}

The original mechanism proposed for rectification of electrical transmission was represented as a simple analogy to an electric rectifier or diode (Furshpan and Potter, 1959), in which separation of negative and positive permanent charges results in an asymmetric energy barrier. This barrier generates instantaneous transjunctional current $\left(I_{j}\right)$ rectification with characteristics of a $\mathrm{p}-\mathrm{n}$ junction in semiconductors. At that time GJ channels had not yet been discovered and thus the properties of the rectifier and the electrostatic effect were assigned to the "synaptic membrane". Nonetheless, the novel hypothesis of $\mathrm{p}-\mathrm{n}$ junctions in biological membranes was examined (Mauro, 1962; Coster, 1965), and provided a theoretical framework for considering fixed charges in junctional membranes (Brink and Dewey, 1980) that could explain the steep rectification of the junctional conductance-voltage relation $\left(g_{\mathrm{j}}-V_{\mathrm{j}}\right)$ in some electrical synapses.

The hypothesis that electrical rectification could arise from an asymmetry in aHC composition came in the late 70's (Bennett, 1977; Loewenstein, 1981). With the exogenous expression of different $\mathrm{Cx}$ isoforms, it was possible to examine this hypothesis. Indeed, electrical rectification of heterotypic GJ channels (originally called heteromolecular or hybrid cell-cell channels) was first studied in pair of oocytes overexpressing $\mathrm{Cx} 32 / \mathrm{Cx} 26$ or Cx32/Cx43 GJs (Swenson et al., 1989; Werner et al., 1989; Barrio et al., 1991). In the case of heterotypic Cx32/Cx26 GJ channels, asymmetries in the instantaneous and steady-state $g_{\mathrm{j}}-V_{\mathrm{j}}$ relationships were observed (Barrio et al., 1991). To make a clear distinction between instantaneous and steady-state asymmetries in the $g_{j}-V_{j}$ relationship, we refer to instantaneous and steadystate asymmetries as "electrical rectification" and "asymmetric gating", respectively.

Based on single GJ channel and $\mathrm{HC}$ recordings showing multiple $I_{\mathrm{j}}$ substates, we know that the steady-state $I_{\mathrm{j}}-V_{\mathrm{j}}$ relationship of GJ channels is the product of two $V_{\mathrm{j}}$-sensitive gating mechanisms present in each aHC, the fast or " $V_{j}$ " gate and the slow or "loop" gate (Bukauskas and Verselis, 2004). The probability of each $V_{\mathrm{j}}$-sensitive gate to dwell in a closed state is a function of the intensity and relative polarity of $V_{j}$ (gating polarity). The instantaneous $I_{j}-V_{j}$ relationship is mostly determined by the electrical properties of the unitary conductance of the fully open state $\left(\gamma_{\mathrm{o}}\right)$ of GJ channels, which can rectify by allowing larger $I_{j} \mathrm{~s}$ in one direction than in the other. The unitary conductance of the residual state ( $\gamma_{\text {res }}$; one or two fast gates in closed position) may also rectify (Bukauskas et al., 1995; Oh et al., 1999), and therefore can contribute to electrical rectification. In general, homotypic GJs show symmetric $g_{j}-V_{j}$ relationships for either polarity of $V_{\mathrm{j}}$ (Figure 3A). However, asymmetry in the composition of aHCs (or transjunctional asymmetry in cytosolic factors; see below) may result in electrical rectification (Figure 3B) and asymmetric gating.

The mechanism for asymmetric gating observed in Cx32/Cx26 GJ channels was explained by a difference in gating polarity of voltage-sensitive gates present in Cx26 and Cx32 aHCs (Verselis et al., 1994). Heterotypic GJs that possess aHC with opposite gating polarity exhibit marked asymmetric gating since one polarity of $V_{\mathrm{j}}$ simultaneously opens the gates in both aHCs, and the opposite polarity closes them. In addition to opposite gating polarity, asymmetric gating can also be produced by differences in unitary conductances of aHCs $\left(\gamma_{\mathrm{o}, \mathrm{H}}\right)$, or simply by differences in intrinsic sensitivity to $V_{\mathrm{j}}$ (Bukauskas et al., 1995; Rackauskas et al., 2007). When $\gamma_{o, H} s$ are considerably dissimilar, like in the case of $\mathrm{Cx} 43 / \mathrm{Cx} 45$ heterotypic GJs $\left(\gamma_{\mathrm{o}, \mathrm{H}}\right.$ of $\mathrm{Cx} 43$ is $\sim 4$ times higher than that of $\mathrm{Cx} 45)$, a bigger fraction of $V_{\mathrm{j}}$ drops across the aHC with higher resistance $(\mathrm{Cx} 45)$, thus enhancing its sensitivity to $V_{\mathrm{j}}$ compared to the aHC with smaller 
A
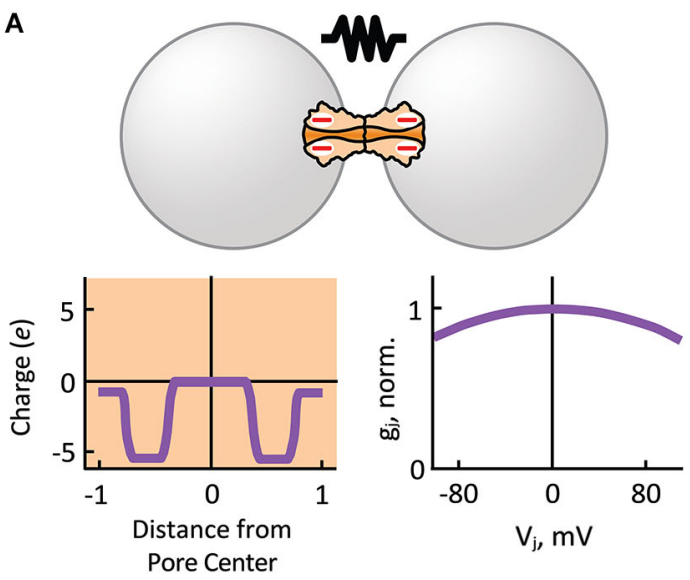

B
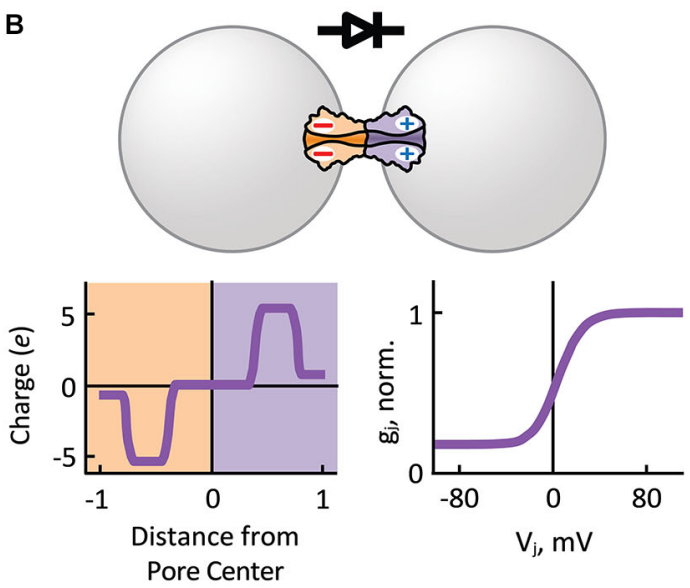

FIGURE 3 | Hemichannel composition determines the symmetry of electrical transmission. (A) Homotypic gap junction channels that comprise symmetric charge distribution with respect to the pore center of the channel, behave as passive resistors with symmetric junctional conductance $\left(g_{j}\right)$ over transjunctional voltage $\left(V_{j}\right)$ dependence (normalized to $g_{\mathrm{j}}$ value at $V_{\mathrm{j}}$ equal zero). (B) Heterotypic gap junction channels that comprise an asymmetry in positive and negative charge distribution with respect to the pore center of the channel behave as electrical rectifiers ( $p-n$ junction) with steep asymmetric $g_{\mathrm{j}}-V_{\mathrm{j}}$ dependence. Normally, depolarizing (positive) potentials are more easily transmitted from the cell with negatively charged $\mathrm{HCs}$ to the cell with positively charged $\mathrm{HCs}$.

resistance (Cx43). Therefore, differential drop of $V_{\mathrm{j}}$ in aHCs of heterotypic GJs may also result in asymmetric gating (Bukauskas et al., 2002). Although asymmetric gating may be important to determine $I_{j}$ and transjunctional flux directionalities under longlasting asymmetries in $V_{\mathbf{j}}$ (Palacios-Prado and Bukauskas, 2009), electrical rectification (instantaneous asymmetry) determines the directionality of synaptic electrical transmission between neurons with brief (millisecond) oscillatory changes in $V_{\mathrm{j}}$ during action potentials.

Analysis of heterotypic Cx32/Cx26 GJs at the single channel level revealed that $\gamma_{0}$ rectifies depending on $V_{\mathrm{j}}$ (Bukauskas et al., 1995). Using this premise, an electrodiffusive model that solves the Poisson-Nernst-Planck (PNP) equations in one dimension (Chen and Eisenberg, 1993) was used to describe the asymmetric single channel fluxes and currents observed in heterotypic Cx32/Cx26 GJs (Oh et al., 1999). The PNP model in combination with site-directed mutagenesis successfully predicted that electrical rectification was produced by an asymmetric position of fixed charged amino acid residues present in the heterotypic Cx32/Cx26 channel pore. These findings demonstrated that the original diode hypothesis of $\mathrm{p}-\mathrm{n}$ junctions could indeed generate electrical rectification of synaptic transmission based on the asymmetric position of charges near the channel-pore surface (Figure 3B) that, in turn, produce differences in ionic conductance and selectivity of HCs (Suchyna et al., 1999). Thus, heterotypic GJ channels that form rectifying junctions with steep asymmetric $g_{j}-V_{j}$ relationship (Figure $4 \mathbf{A}$ ) can make an electrical synapse nearly unidirectional by allowing the transmission of depolarizing and hyperpolarizing potentials in only one direction (opposite to each other), and restricting the transmission of depolarizing and hyperpolarizing potentials in the opposite direction (Figure 4B). The mechanism for electrical rectification and asymmetric gating observed in the Drosophila GFS is indeed associated with molecular asymmetry of HCs (Phelan et al., 2008). Since ShakB(Neural+16) and ShakB(Lethal) variants exhibit significant differences in amino acid sequence and sensitivity to $V_{\mathrm{j}}$, it is likely that electrical rectification arises from asymmetry in position of charges (p-n junction), and asymmetric gating arises from differences in intrinsic $V_{\mathrm{j}}$-sensitivity of aHCs rather than opposite gating polarities or differences in $\gamma_{\mathrm{o}, \mathrm{H}}$.

In addition to the p-n junction hypothesis for electrical rectification, a unique voltage-dependent gating mechanism was proposed after a detailed characterization of the rectifying crayfish giant motor synapse using high-quality voltage clamp at low temperatures (Jaslove and Brink, 1986). These studies suggested that, rather than an instantaneous electrostatic effect, the rectification profile of the $I_{\mathrm{j}}-V_{\mathrm{j}}$ relationship contained a voltagedependent kinetic component with a time constant in the order of milliseconds, which was attributed to a rapid gating mechanism present in one of the aHCs. The authors proposed that this gate was set to a low open probability at resting conditions and that changing the polarity of $V_{\mathrm{j}}$ would rapidly open the gates. This "millisecond timescale" gating mechanism has not been reported in any other $\mathrm{Cx}$ - or Inx-based rectifying electrical synapse; hence it is unclear whether rapid gating mechanisms may contribute to the observed electrical rectification in other invertebrate and vertebrate electrical synapses.

Gap junctions can occur in homocellular or heterocellular junctions; that is, coupled cells can be from the same or different cell types and perform similar or different functions, respectively. One remarkable similarity among electrical synapses showing steep rectification is that they occur mostly in heterocellular junctions and very often there is a difference in the resting potential of coupled neurons that give rise to a relatively constant $V_{\mathrm{j}}$ (Giaume and Korn, 1983; Ramón and Rivera, 1986). Regardless of the mechanism of rectification, the resting $V_{j}$ derived from the difference in the resting potential of neurons forming rectifying electrical synapses in crayfish and leech is essential to produce steep rectification, since bidirectional transmission of depolarization pulses could be achieved by reversing the resting 
A
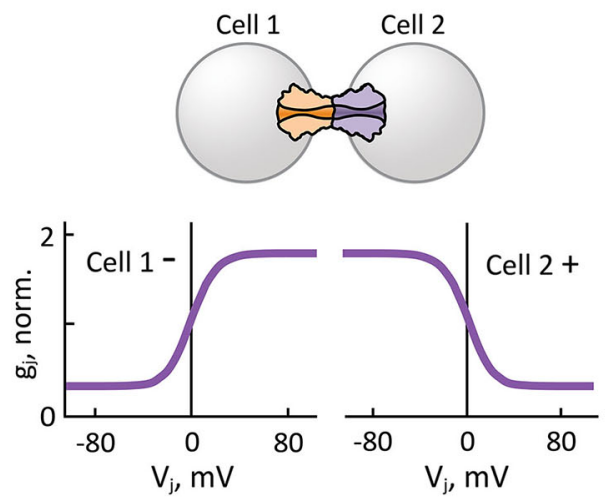

B
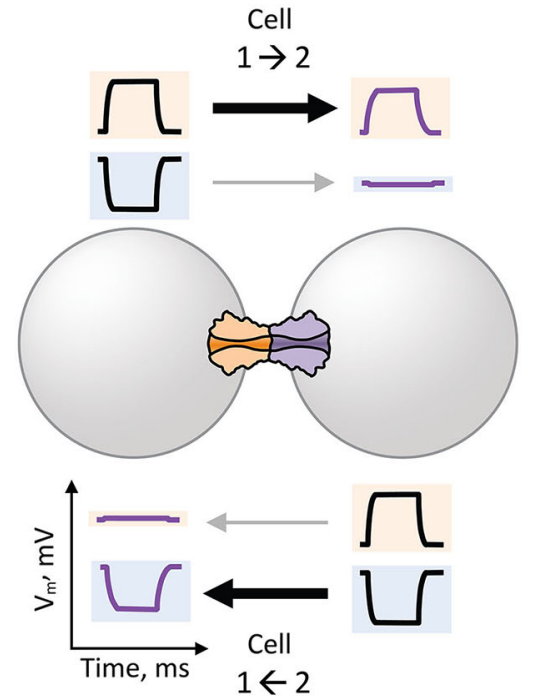

FIGURE 4 | Hemichannel composition and intercellular gradient of charged cytosolic factors can lead to rectification of electrical transmission. (A,B) Heterotypic gap junction channels with steep asymmetric $g_{j}-V_{j}$ dependence (A) facilitate or attenuate the electrical transmission of depolarizing (positive) potentials from Cell 1 to Cell $2(1 \rightarrow 2)$ or $1 \leftarrow 2$, respectively (B). The same junctions facilitate or attenuate the electrical transmission of hyperpolarizing (negative) potentials from $1 \leftarrow 2$ or
C $\left[\mathrm{Mg}^{2+}\right]_{1}>\left[\mathrm{Mg}^{2+}\right]_{2}$
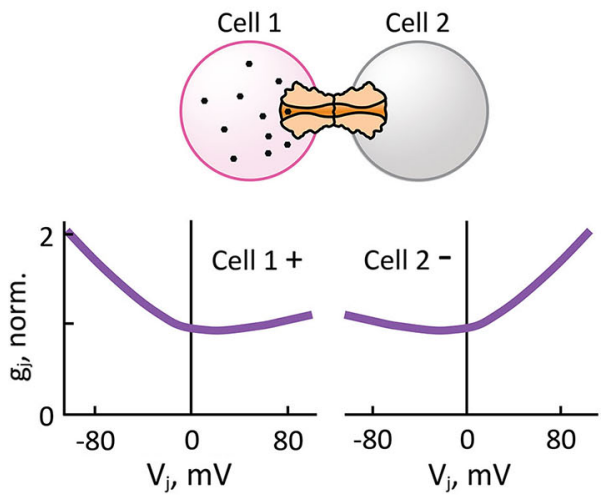

D

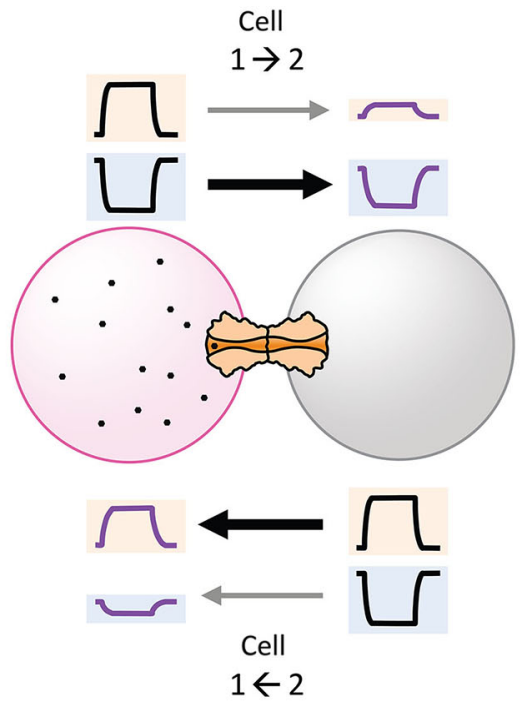

$1 \rightarrow 2$, respectively (B). (C, D) Transjunctional gradient of free magnesium ion concentration $\left(\left[\mathrm{Mg}^{2+}\right]_{i}\right)$ induces asymmetric $g_{j}-V_{j}$ dependence in homotypic gap junction channels (C) that are hypersensitive to $\left[\mathrm{Mg}^{2+}\right]_{i}$, such as $\mathrm{C} \times 36$ gap junction channels. Electrical transmission of depolarizing potentials is facilitated from $2 \rightarrow 1$ (D), which is the opposite direction of the transjunctional $\left[\mathrm{Mg}^{2+}\right]_{i}$ gradient $(1 \rightarrow 2)$. The same transjunctional $\left[\mathrm{Mg}^{2+}\right]_{i}$ gradient facilitates the electrical transmission of hyperpolarizing potentials from $1 \rightarrow 2$ (D).

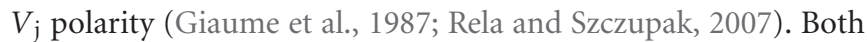
p-n junction and rapid gating mechanisms imply a molecular asymmetry in aHC composition, and both require a resting $V_{\dot{j}}$ (difference in membrane potentials between the coupled cells) to exhibit significant electrical rectification or asymmetric gating, respectively. As an analogy to $\mathrm{p}-\mathrm{n}$ junctions in semiconductors and silica nanochannels (Cheng and Guo, 2007), the resting $V_{\mathrm{j}}$ would normally set GJs to a low conductive state by producing a "reversed bias" effect (expression used when the flow of current is obstructed by increasing the resistance). Only action potentials that lower this resting $V_{j}$ would produce a "forward bias" effect in the junction to allow the spread of electrotonic potentials.

\section{CONTRIBUTION OF INTRACELLULAR SOLUBLE FACTORS}

Gap junction channels and HCs are highly regulated according to cellular requirements and respond to various changes in the extracellular and intracellular environments. Besides their sensitivity to $V_{\mathrm{j}}$, GJ channels and HCs are sensitive to phosphorylation, lipophilic molecules and other chemical factors (Baldridge et al., 1987; Bennett et al., 1991; Harris, 2001; Bukauskas and Verselis, 2004; Jouhou et al., 2007; Márquez-Rosado et al., 2012). Furthermore, GJ channels are sensitive to changes in intracellular ionic composition, such as intracellular $\mathrm{pH}, \mathrm{Mg}^{2+}$ and $\mathrm{Ca}^{2+}$ that may vary under physiological conditions (Noma and Tsuboi, 1987; Cheng and Reynolds, 2000; Chesler, 2003; Matsuda et al., 2010; Shindo et al., 2010; Yamanaka et al., 2013). This suggests that 
modulation of electrical and metabolic gap junctional intercellular communication by these factors may be important for normal cell function.

It has been recently reported that $\mathrm{Cx} 36$-containing electrical synapses expressed in the mesencephalic nucleus of the trigeminal nerve (MesV) and the thalamic reticular nucleus (TRN) as well as heterologous expression systems transfected with Cx36 are bi-directionally modulated by changes in intracellular concentration of free $\mathrm{Mg}^{2+}\left(\left[\mathrm{Mg}^{2+}\right]_{i}\right)$ (Palacios-Prado et al., 2013, 2014). This is a novel $\mathrm{Mg}^{2+}$-dependent form of electrical synaptic plasticity where $g_{j}$ can be augmented or reduced by lowering or increasing $\left[\mathrm{Mg}^{2+}\right]_{i}$, respectively. These studies support the notion that $\left[\mathrm{Mg}^{2+}\right]_{\mathrm{i}}$ controls neuronal coupling via modulation of gating mechanisms of Cx36 GJs by interacting with a $\mathrm{Mg}^{2+}$ sensitive domain located in the lumen of the GJ channel. Since intracellular levels of ATP determines $\left[\mathrm{Mg}^{2+}\right]_{\mathrm{i}}$ (Lüthi et al., 1999), $\mathrm{Mg}^{2+}$-dependent plasticity of electrical synapses could be under control of neuronal metabolism and circadian rhythms (Dworak et al., 2010). In addition, electrical synaptic transmission could potentially decrease after neuronal depolarization and glutamate exposure, due to an increment in $\left[\mathrm{Mg}^{2+}\right]_{\mathrm{i}}$ (Kato et al., 1998; Shindo et al., 2010).

Electrical synapses formed by $\mathrm{Cx} 36$ show a unique $\mathrm{Mg}^{2+}$ dependent instantaneous $g_{\mathfrak{j}}-V_{\mathfrak{j}}$ relationship, in which instantaneous $g_{\mathrm{j}}$ increases over $V_{\mathrm{j}}$ under high $\left[\mathrm{Mg}^{2+}\right]_{\mathrm{i}}$, or remain constant over $V_{\mathrm{j}}$ under low $\left[\mathrm{Mg}^{2+}\right]_{\mathrm{i}}$. Interestingly, an intercellular gradient of $\mathrm{Mg}^{2+}$ (asymmetric transjunctional $\left[\mathrm{Mg}^{2+}\right]_{\mathrm{i}}$ ) produces electrical rectification (Figure $4 \mathrm{C}$ ) and asymmetric gating in homotypic GJs by affecting the instantaneous and steady-state $g_{\mathrm{j}}-V_{\mathrm{j}}$ relationship of $\mathrm{Cx} 36$, respectively (Palacios-Prado et al., 2013, 2014). Asymmetric transjunctional $\left[\mathrm{Mg}^{2+}\right]_{\mathrm{i}}$ produces greater transmission of depolarizing or hyperpolarizing potentials from the cell with lower or higher $\left[\mathrm{Mg}^{2+}\right]_{i}$, respectively, compared to the opposite directions (Figure 4D). To explain this unique electrical rectification of $\mathrm{Cx} 36$, the authors proposed that a combination of two or more mechanisms are necessary: asymmetric fixed charges inside the $\mathrm{Cx} 36 \mathrm{aHC}$ pore that produce a $\mathrm{p}-\mathrm{n}$ junction type of rectification; and a $V_{\mathrm{j}}$-dependent modulation of $\mathrm{Mg}^{2+}$ interaction with its binding sites inside the pore. $\mathrm{Mg}^{2+}$ dependent plasticity of Cx36 GJ channel properties is the only described mechanism so far by which transjunctional asymmetry is derived from a diffusible cytosolic factor that produces electrical rectification in homotypic GJs; all other examples arise from a molecular asymmetry in aHC composition. In principle, transjunctional asymmetry in ATP concentration may also induce electrical rectification by producing a transjunctional asymmetry in $\left[\mathrm{Mg}^{2+}\right]_{\mathrm{i}}$. It is noteworthy that other intracellular diffusible cations such as $\mathrm{H}^{+}, \mathrm{Ca}^{2+}$ and spermine have been shown to affect cell-cell coupling via gating mechanisms in a Cx-specific manner (White et al., 1990; Musa et al., 2004; Harris and Contreras, 2014), but their effect on electrical rectification is yet to be demonstrated.

Asymmetry in the molecular composition of aHCs can also play a role in the effects of cytosolic factors. Heterotypic channels formed by expression of Cx35 and Cx34.7 in cell lines (the Cxs present at Club ending-Mauthner cell synapses) exhibited differential sensitivity to changes in $\left[\mathrm{Mg}^{2+}\right]_{\mathrm{i}}$, suggesting that molecular differences in heterotypic junctions might also contribute to generate electrical rectification by expressing a differential sensitivity to cytosolic factors (Rash et al., 2013).

\section{FUNCTIONAL PROPERTIES OF RECTIFYING ELECTRICAL SYNAPSES}

Rectifying electrical synapses have been proposed to play important functional roles within various neuronal networks (Furshpan and Potter, 1957; Edwards et al., 1999; Allen et al., 2006; Gutierrez and Marder, 2013). Providing directionality to electrical transmission between pre- and postsynaptic neurons, rectifying electrical synapses can significantly contribute to general signal transduction as in C. elegans (Starich et al., 2009) and are a feature in many escape networks (Furshpan and Potter, 1959; Edwards et al., 1999; Allen et al., 2006; Phelan et al., 2008). Rectifying electrical synapses were initially described at the giant motor synapses of the abdominal nerve cord of the crayfish between GFs and giant motor axons that innervate the flexor musculature of the tail (Furshpan and Potter, 1959). They also mediate directional communication in the Drosophila GFS (Allen et al., 2006) and between mechanoreceptor afferents and interneurons synapsing on the lateral giant neurons in crayfish (Edwards et al., 1999). Their ability to generate voltage-dependent directional transmission was also reported to be advantageous for certain motor behaviors in leech (Rela and Szczupak, 2003) and in fish spinal cord (Auerbach and Bennett, 1969), providing fast directional communication between identifiable interneurons and motor neurons.

Interestingly, rectifying electrical synapses can also underlie bidirectional communication between neuronal processes of dissimilar size, compensating for unfavorable electrical and geometrical conditions for the symmetrical spread of currents through the junctions. This is the case of a group of identifiable auditory synapses on the Mauthner cell known as Club endings (Pereda et al., 2004); the Mauthner cell network mediates auditoryevoked escape responses in fish (Faber and Pereda, 2011). Because electrical synapses at Club endings are bidirectional, the signals produced by a population of active Club endings in the Mauthner cell dendrite can influence the excitability of nonactive neighboring Club endings, thus serving as a mechanism for "lateral excitation" (Pereda et al., 1995). Lateral excitation increases the sensitivity of sensory inputs (Herberholz et al., 2002). Electrical rectification favors this mechanism of lateral excitation by promoting the spread of currents originated in the dendrite to the presynaptic afferents, which otherwise would passively spread towards the lower input resistance soma of the Mauthner cell (Rash et al., 2013). Thus, by favoring the spread of currents to the presynaptic afferent, the rectification properties of electrical synapses between Club endings and the Mauthner cell enhance bi-directionality of electrical communication between these two cells of dissimilar size and geometry. From the functional point of view, lateral excitation promotes the coordinated activity of a population of Club endings, thus increasing the efficacy of the auditory input for the initiation of an escape response. 


\section{A}

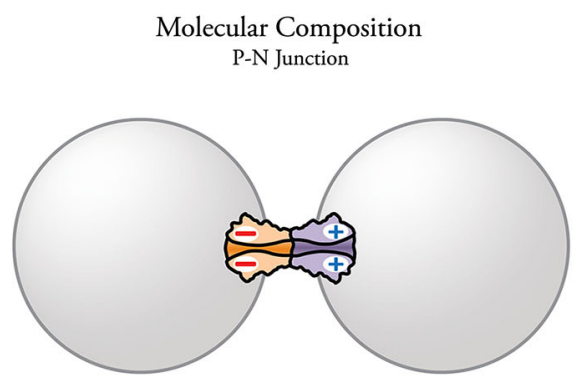

B

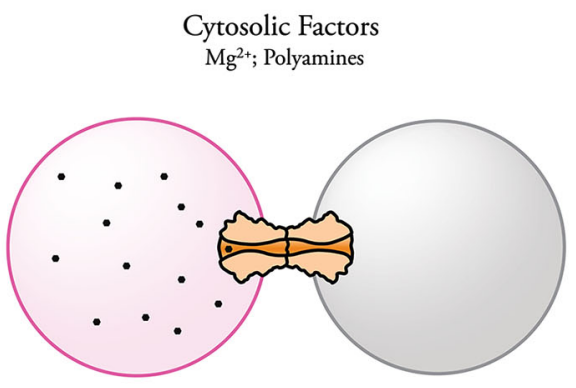

C

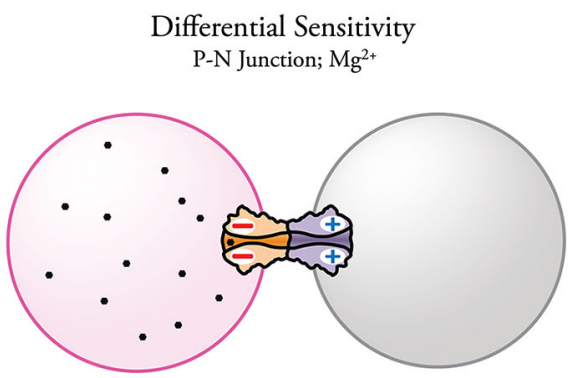

FIGURE 5 | Possible mechanisms underlying electrical rectification at electrical synapses. (A) Steep electrical rectification can be attainable by the separation of fixed positive and negative charges at opposite ends of heterotypic gap junction channels ( $p$-n junction) resulting from asymmetries in the molecular composition of the HCs. (B) Electrical rectification can also result from the presence of an intercellular gradient of charged cytosolic factors, such as $\mathrm{Mg}^{2+}$ and spermine, which alter channel conductance. Molecular diversity can make some channels more susceptible of interacting with certain cytosolic factors. (C) Complex electrical rectification can arise from the combination of both mechanisms.

Recent studies suggest that rectifying electrical synapses are capable on endowing networks with more complex behaviors. Modeling studies explored the impact of rectifying electrical synapses in a pattern-generating neuronal network containing both chemical and electrical synapses (Gutierrez and Marder, 2013). The presence of rectifying electrical synapses was observed to have profound functional consequences, altering the sensitivity of the network dynamics to variations in the strength of chemical synapses (Gutierrez and Marder, 2013). Remarkably, the addition of rectifying electrical synapses to certain network configurations yielded robust circuit dynamics that were insensitive to variations in the strength of chemical synapses (Gutierrez and Marder,
2013), suggesting that the presence of rectifying electrical synapses is likely to play important roles in the stability and function of neural networks.

Finally, as a result on their voltage-dependent properties, rectifying electrical synapses were proposed to act as coincidence detectors (Edwards et al., 1998; Marder, 2009). Coincidence detection is an essential property of all nervous systems and is sustained by a variety of molecular, cellular and network properties. This phenomenon has been implicated in visual perception (Veruki and Hartveit, 2002), sound source localization (Joris et al., 1998), memory formation (Tsien, 2000), and motor control (Hjorth et al., 2009), amongst others. While nonrectifying electrical synapses are considered coincidence detectors of inputs arriving simultaneously at two different coupled neurons (Galarreta and Hestrin, 2001; Veruki and Hartveit, 2002), electrical rectification underlies the ability of the lateral giant neurons of crayfish to sum inputs that arrive synchronously (Edwards et al., 1998). Remarkably, this mechanism provides a significant temporal fidelity and it does not operate for inputs that are separated by only $100 \mathrm{~ms}$ or more. Because rectifying synapses in this system only allow bidirectional current flow when the presynaptic afferents are depolarized relative to the postsynaptic compartment (the lateral giant neuron), current flows increase during the presynaptic spike and remain electrically coupled after its completion (Edwards et al., 1998). Taking advantage of this property, synchronous inputs from mechanoreceptor afferents and interneurons integrate effectively and produce large excitatory responses. Asynchronous inputs, on the other hand, are much less efficient in activating the mechanism because: (1) the early arriving postsynaptic potential retards the opening of voltage-sensitive channels at additional synapses; and (2) the late arriving synaptic currents are shunted by the increase in $g_{j}$. Given the involvement of these neurons in escape responses, the coincidence detection mediated by the voltage-dependent properties of rectifying electrical synapses allows crayfish to elicit reflex escape responses only to particularly abrupt mechanical stimuli (Edwards et al., 1998).

\section{CONCLUSIONS}

Electrical transmission has become a topic of high interest in neuroscience. Together with the already established role of electrical synapses in invertebrates and cold-blooded vertebrates, evidence for the presence and importance of electrical synapses in the diverse areas of the mammalian brain continues to increase. Despite their wide distribution and functional relevance, the molecular complexity of electrical synapses and how this complexity affects synaptic function is still poorly understood. The evidence reviewed here indicates that the molecular composition of each aHC can endow neuronal GJs with important functional properties. More specifically, asymmetry in the molecular composition of aHCs has been associated with rectification of electrical transmission. The fact that such association was found at both Inx- and Cx-based electrical synapses emphasizes the contribution of the molecular asymmetry in underlying this voltage-dependent phenomenon. It has been shown that heterotypic channels with asymmetric position of charges near 
the channel-pore surface act as p-n junctions (diode hypothesis) with asymmetric transjunctional current flow (Figure 5A). Electrical rectification can also be observed at homotypic channels, arising from transjunctional asymmetries in the concentration of cytosolic factors that are capable of interacting with the channel pore (Figure 5B). Finally, cytosolic factors can contribute to electrical rectification at heterotypic junctions if one of the aHCs is more susceptible to interact with them, further enhancing the rectifying properties of the junction (Figure 5C).

Despite the presence of molecularly distinct pre- and postsynaptic sites, chemical synapses are considered indivisible functional units at which both sites are required to generate synaptic function. Electrical synapses can be viewed in a similar way. The fact that the docking of two $\mathrm{HC}$ is required does not necessarily imply that their molecular composition and that of the hemiplaques are the same. Hemiplaques should be different, suggesting that electrical synapses in analogy to chemical synapses can have distinct pre- and postsynaptic sites, endowing electrical synapses with more complex functional properties. While we emphasize in this article asymmetries in the composition of aHCs by GJ-forming proteins, asymmetries might also include the presence of associated scaffolding and regulatory proteins. Finally, an interesting scenario would be if asymmetries could be dynamically created by posttranslational modifications of Cxs in only one of the aHCs (asymmetric phosphorylation), or by differences in the intracellular concentration of soluble factors that affect channels properties as a result of metabolic changes in one of the coupled cells, providing electrical synapses with plastic rectifying properties.

\section{ACKNOWLEDGMENTS}

Portions of this work have been presented as part of a thesis dissertation (Nicolás Palacios-Prado). The authors are indebted to the Rainbow Kittens. Supported by the Grass Foundation, a Howard Hughes Medical Institute International Student Research Fellowship to Nicolás Palacios-Prado, a Marie-Curie Zukunftskolleg Incoming Fellowship to Wolf Huetteroth, and National Institutes of Health grants NIH DC03186, DC011099, NS055726, NS085772 and NS0552827 to Alberto E. Pereda.

\section{REFERENCES}

Abascal, F., and Zardoya, R. (2013). Evolutionary analyses of gap junction protein families. Biochim. Biophys. Acta 1828, 4-14. doi: 10.1016/j.bbamem.2012.02.007 Adams, M. D., Celniker, S. E., Holt, R. A., Evans, C. A., Gocayne, J. D., Amanatides, P. G., et al. (2000). The genome sequence of Drosophila melanogaster. Science 287, 2185-2195. doi: 10.1126/science.287.5461.2185

Allen, M. J., Godenschwege, T. A., Tanouye, M. A., and Phelan, P. (2006). Making an escape: development and function of the Drosophila giant fibre system. Semin. Cell Dev. Biol. 17, 31-41. doi: 10.1016/j.semcdb.2005.11.011

Altun, Z. F., Chen, B., Wang, Z. W., and Hall, D. H. (2009). High resolution map of Caenorhabditis elegans gap junction proteins. Dev. Dyn. 238, 1936-1950. doi: 10. 1002/dvdy.22025

Apostolides, P. F., and Trussell, L. O. (2013). Regulation of interneuron excitability by gap junction coupling with principal cells. Nat. Neurosci. 16, 1764-1772. doi: 10.1038/nn.3569

Auerbach, A. A., and Bennett, M. V. L. (1969). A rectifying electrotonic synapse in the central nervous system of a vertebrate. J. Gen. Physiol. 53, 211-237. doi: 10. 1085/jgp.53.2.211
Baldridge, W. H., Ball, A. K., and Miller, R. G. (1987). Dopaminergic regulation of horizontal cell gap junction particle density in goldfish retina. J. Comp. Neurol. 265, 428-436. doi: 10.1002/cne.902650310

Barnes, T. M., and Hekimi, S. (1997). The Caenorhabditis elegans avermectin resistance and anesthetic response gene unc- 9 encodes a member of a protein family implicated in electrical coupling of excitable cells. J. Neurochem. 69, 2251-2260. doi: 10.1046/j.1471-4159.1997.69062251.x

Barrio, L. C., Suchyna, T., Bargiello, T., Xu, L. X., Roginski, R. S., Bennett, M. V., et al. (1991). Gap junctions formed by connexins 26 and 32 alone and in combination are differently affected by applied voltage. Proc. Natl. Acad. Sci. U S A 88, 8410-8414. doi: 10.1073/pnas.88.19.8410

Baylor, D. A., and Nicholls, J. G. (1969). Chemical and electrical synaptic connexions between cutaneous mechanoreceptor neurones in the central nervous system of the leech. J. Physiol. 203, 591-609.

Bennett, M. V. L. (1977). "Electrical transmission: a functional analysis and comparison to chemical transmission," in The Handbook of Physiology, ed E. Kandel (Washington: American Physiological Society), 357-416.

Bennett, M. V., Barrio, L. C., Bargiello, T. A., Spray, D. C., Hertzberg, E., and Sáez, J. C. (1991). Gap junctions: new tools, new answers, new questions. Neuron 6 , 305-320. doi: 10.1016/0896-6273(91)90241-q

Bennett, M. V. L., Contreras, J. E., Bukauskas, F. F., and Sáez, J. C. (2003). New roles for astrocytes: gap junction hemichannels have something to communicate. Trends Neurosci. 26, 610-617. doi: 10.1016/j.tins.2003.09.008

Bennett, M. V. L., and Zukin, R. S. (2004). Electrical coupling and neuronal synchronization in the Mammalian brain. Neuron 41, 495-511. doi: 10.1016/s08966273(04)00043-1

Blagburn, J. M., Alexopoulos, H., Davies, J. A., and Bacon, J. P. (1999). Null mutation in shaking-B eliminates electrical, but not chemical, synapses in the Drosophila giant fiber system: a structural study. J. Comp. Neurol. 404, 449-458. doi: 10.1002/(sici)1096-9861(19990222)404:4<449::aid-cne3>3.3.co;2-4

Brink, P. R., and Dewey, M. M. (1980). Evidence for fixed charge in the nexus. Nature 285, 101-102. doi: 10.1038/285101a0

Bruzzone, R., Hormuzdi, S. G., Barbe, M. T., Herb, A., and Monyer, H. (2003). Pannexins, a family of gap junction proteins expressed in brain. Proc. Natl. Acad. Sci. US A 11, 13644-13649. doi: 10.1073/pnas.2233464100

Bukauskas, F. F., Bukauskiene, A., Verselis, V. K., and Bennett, M. V. L. (2002). Coupling asymmetry of heterotypic connexin 45/connexin 43-EGFP gap junctions: properties of fast and slow gating mechanisms. Proc. Natl. Acad. Sci. U S A 99, 7113-7118. doi: 10.1073/pnas.032062099

Bukauskas, F. F., Elfgang, C., Willecke, K., and Weingart, R. (1995). Heterotypic gap junction channels (connexin26-connexin32) violate the paradigm of unitary conductance. Pflugers Arch. 429, 870-872. doi: 10.1007/bf00374812

Bukauskas, F. F., and Verselis, V. K. (2004). Gap junction channel gating. Biochim. Biophys. Acta 1662, 42-60. doi: 10.1016/j.bbamem.2004.01.008

Chen, D. P., and Eisenberg, R. S. (1993). Flux, coupling and selectivity in ionic channels of one conformation. Biophys. J. 65, 727-746. doi: 10.1016/s00063495(93)81099-3

Cheng, L.-J., and Guo, L. J. (2007). Rectified ion transport through concentration gradient in homogeneous silica nanochannels. Nano Lett. 7, 3165-3171. doi: 10. 1021/nl071770c

Cheng, C., and Reynolds, I. J. (2000). Subcellular localization of glutamatestimulated intracellular magnesium concentration changes in cultured rat forebrain neurons using confocal microscopy. Neuroscience 95, 973-979. doi: 10. 1016/s0306-4522(99)00471-6

Chesler, M. (2003). Regulation and modulation of pH in the brain. Physiol. Rev. 83, 1183-1221. doi: 10.1152/physrev.00010.2003

Chou, Y.-H., Spletter, M. L., Yaksi, E., Leong, J. C. S., Wilson, R. I., and Luo, L. (2010). Diversity and wiring variability of olfactory local interneurons in the Drosophila antennal lobe. Nat. Neurosci. 13, 439-449. doi: 10.1038/nn. 2489

Condorelli, D. F., Belluardo, N., Trovato-Salinaro, A., and Mudò, G. (2000). Expression of Cx36 in mammalian neurons. Brain Res. Brain Res. Rev. 32, 72-85. doi: 10.1016/s0165-0173(99)00068-5

Connors, B. W., and Long, M. A. (2004). Electrical synapses in the mammalian brain. Annu. Rev. Neurosci. 27, 393-418. doi: 10.1146/annurev.neuro.26.041002. 131128

Contreras, J. E., Sánchez, H. A., Eugenín, E. A., Speidel, D., Theis, M., Willecke, K., et al. (2002). Metabolic inhibition induces opening of unapposed connexin 43 gap junction hemichannels and reduces gap junctional communication in 
cortical astrocytes in culture. Proc. Natl. Acad. Sci. U S A 99, 495-500. doi: 10. 1073/pnas.012589799

Coster, H. G. (1965). A quantitative analysis of the voltage-current relationships of fixed charge membranes and the associated property of "punch-through". Biophys. J. 5, 669-686. doi: 10.1016/s0006-3495(65)86745-5

Curtin, K. D., Zhang, Z., and Wyman, R. J. (2002). Gap junction proteins expressed during development are required for adult neural function in the Drosophila optic lamina. J. Neurosci. 22, 7088-7096.

Dahl, G., and Locovei, S. (2006). Pannexin: to gap or not to gap, is that a question? IUBMB Life 58, 409-419. doi: 10.1080/15216540600794526

Dahl, G., and Muller, K. J. (2014). Innexin and pannexin channels and their signaling. FEBS Lett. 588, 1396-1402. doi: 10.1016/j.febslet.2014.03.007

Das, A., Chiang, A., Davla, S., Priya, R., Reichert, H., Vijayraghavan, K., et al. (2011). Identification and analysis of a glutamatergic local interneuron lineage in the adult Drosophila olfactory system. Neural Syst. Circuits 1:4. doi: 10. 1186/2042-1001-1-4

Devor, A., and Yarom, Y. (2002). Electrotonic coupling in the inferior olivary nucleus revealed by simultaneous double patch recordings. J. Neurophysiol. 87, 3048-3058.

Dworak, M., McCarley, R. W., Kim, T., Kalinchuk, A. V., and Basheer, R. (2010). Sleep and brain energy levels: ATP changes during sleep. J. Neurosci. 30, 90079016. doi: 10.1523/JNEUROSCI.1423-10.2010

Eastman, S. D., Chen, T. H.-P., Falk, M. M., Mendelson, T. C., and Iovine, M. K. (2006). Phylogenetic analysis of three complete gap junction gene families reveals lineage-specific duplications and highly supported gene classes. Genomics 87, 265-274. doi: 10.1016/j.ygeno.2005.10.005

Edwards, D. H., Heitler, W. J., and Krasne, F. B. (1999). Fifty years of a command neuron: the neurobiology of escape behavior in the crayfish. Trends Neurosci. 22, 153-161. doi: 10.1016/s0166-2236(98)01340-x

Edwards, D. H., Yeh, S.-R., and Krasne, F. B. (1998). Neuronal coincidence detection by voltage-sensitive electrical synapses. Proc. Natl. Acad. Sci. U S A 95, 71457150. doi: 10.1073/pnas.95.12.7145

Faber, D. S., and Pereda, A. E. (2011). "Physiology of the mauthner cell: function", in Encyclopedia of Fish Physiology: From Genome to Environment, vol. 1, ed A. P. Farrell (San Diego: Academic Press), 73-79.

Furshpan, E. J., and Potter, D. D. (1957). Mechanism of nerve-impulse transmission at a crayfish synapse. Nature 180, 342-343. doi: 10.1038/180342a0

Furshpan, E. J., and Potter, D. D. (1959). Transmission at the giant motor synapses of the crayfish. J. Physiol. 145, 289-325.

Galarreta, M., and Hestrin, S. (2001). Spike transmission and synchrony detection in networks of GABAergic interneurons. Science 292, 2295-2299. doi: 10. 1126/science. 1061395

Ganfornina, M. D., Sánchez, D., Herrera, M., and Bastiani, M. J. (1999). Developmental expression and molecular characterization of two gap junction channel proteins expressed during embryogenesis in the grasshopper Schistocerca americana. Dev. Genet. 24, 137-150. doi: 10.1002/(sici)15206408(1999) 24:1/2<137::aid-dvg13>3.3.co;2-z

Giaume, C., Kado, R. T., and Korn, H. (1987). Voltage-clamp analysis of a crayfish rectifying synapse. J. Physiol. 386, 91-112.

Giaume, C., and Korn, H. (1983). Bidirectional transmission at the rectifying electrotonic synapse: a voltage-dependent process. Science 220, 84-87. doi: 10. $1126 /$ science.6298940

Goodenough, D. A., and Paul, D. L. (2009). Gap junctions. Cold Spring Harb. Perspect. Biol. 1:a002576. doi: 10.1101/cshperspect.a002576

Gutierrez, G. J., and Marder, E. (2013). Rectifying electrical synapses can affect the influence of synaptic modulation on output pattern robustness. J. Neurosci. 33, 13238-13248. doi: 10.1523/JNEUROSCI.0937-13.2013

Haas, J. S., Zavala, B., and Landisman, C. E. (2011). Activity-dependent long-term depression of electrical synapses. Science 334, 389-393. doi: 10.1126/science. 1207502

Harris, A. L. (2001). Emerging issues of connexin channels: biophysics fills the gap. Q. Rev. Biophys. 34, 325-427. doi: 10.1017/s0033583501003705

Harris, A. L., and Contreras, J. E. (2014). Motifs in the permeation pathway of connexin channels mediate voltage and $\mathrm{Ca}(2+)$ sensing. Front. Physiol. 5:113. doi: 10.3389/fphys.2014.00113

Hasegawa, D. K., and Turnbull, M. W. (2014). Recent findings in evolution and function of insect innexins. FEBS Lett. 588, 1403-1410. doi: 10.1016/j.febslet. 2014.03.006
Herberholz, J., Antonsen, B. L., and Edwards, D. H. (2002). A lateral excitatory network in the escape circuit of crayfish. J. Neurosci. 22, 9078-9085.

Hervé, J. C., Phelan, P., Bruzzone, R., and White, T. W. (2005). Connexins, innexins and pannexins: bridging the communication gap. Biochim. Biophys. Acta 1719, 3-5. doi: 10.1016/j.bbamem.2005.11.013

Hjorth, J., Blackwell, K. T., and Kotaleski, J. H. (2009). Gap junctions between striatal fast-spiking interneurons regulate spiking activity and synchronization as a function of cortical activity. J. Neurosci. 29, 5276-5286. doi: 10. 1523/JNEUROSCI.6031-08.2009

Huang, J., Zhang, W., Qiao, W., Hu, A., and Wang, Z. (2010). Functional connectivity and selective odor responses of excitatory local interneurons in Drosophila antennal lobe. Neuron 67, 1021-1033. doi: 10.1016/j.neuron.2010. 08.025

Iglesias, R., Dahl, G., Qiu, F., Spray, D. C., and Scemes, E. (2009). Pannexin 1: the molecular substrate of astrocyte "hemichannels". J. Neurosci. 29, 7092-7097. doi: 10.1523/JNEUROSCI.6062-08.2009

Jaslove, S. W., and Brink, P. R. (1986). The mechanism of rectification at the electrotonic motor giant synapse of the crayfish. Nature 323, 63-65. doi: 10 . 1038/323063a0

Joris, P. X., Smith, P. H., and Yin, T. C. (1998). Coincidence detection in the auditory system. Neuron 21, 1235-1238. doi: 10.1016/s0896-6273(00)80643-1

Jouhou, H., Yamamoto, K., Iwasaki, M., and Yamada, M. (2007). Acidification decouples gap junctions but enlarges the receptive field size of horizontal cells in carp retina. Neurosci. Res. 57, 203-209. doi: 10.1016/j.neures.2006. 10.006

Kato, H., Gotoh, H., Kajikawa, M., and Suto, K. (1998). Depolarization triggers intracellular magnesium surge in cultured dorsal root ganglion neurons. Brain Res. 779, 329-333. doi: 10.1016/s0006-8993(97)01232-8

Lee, P.-T., Lin, H.-W., Chang, Y.-H., Fu, T.-F., Dubnau, J., Hirsh, J., et al. (2011). Serotonin-mushroom body circuit modulating the formation of anesthesiaresistant memory in Drosophila. Proc. Natl. Acad. Sci. U S A 108, 13794-13799. doi: 10.1073/pnas.1019483108

Li, X., Kamasawa, N., Ciolofan, C., Olson, C. O., Lu, S., Davidson, K. G., et al. (2008). Connexin45-containing neuronal gap junctions in rodent retina also contain connexin36 in both apposing hemiplaques, forming bihomotypic gap junctions, with scaffolding contributed by zonula occludens-1. J. Neurosci. 28, 9769-9789. doi: 10.1523/JNEUROSCI.2137-08.2008

Li, X., Olson, C., Lu, S., Kamasawa, N., Yasumura, T., Rash, J. E., et al. (2004). Neuronal connexin36 association with zonula occludens-1 protein (ZO-1) in mouse brain and interaction with the first PDZ domain of ZO-1. Eur. J. Neurosci. 19, 2132-2146. doi: 10.1111/j.0953-816x.2004.03283.x

Lin, A. C., Bygrave, A. M., de Calignon, A., Lee, T., and Miesenböck, G. (2014). Sparse, decorrelated odor coding in the mushroom body enhances learned odor discrimination. Nat. Neurosci. 17, 559-568. doi: 10.1038/nn.3660

Liu, X., and Davis, R. L. (2009). The GABAergic anterior paired lateral neuron suppresses and is suppressed by olfactory learning. Nat. Neurosci. 12, 53-59. doi: $10.1038 / n n .2235$

Loewenstein, W. R. (1981). Junctional intercellular communication: the cell-to-cell membrane channel. Physiol. Rev. 61, 829-913.

Lüthi, D., Günzel, D., and McGuigan, J. A. (1999). Mg-ATP binding: its modification by spermine, the relevance to cytosolic $\mathrm{Mg} 2+$ buffering, changes in the intracellular ionized $\mathrm{Mg} 2+$ concentration and the estimation of Mg2+ by 31P-NMR. Exp. Physiol. 84, 231-252. doi: 10.1111/j.1469-445x.1999. 01799.x

Macosko, E. Z., Pokala, N., Feinberg, E. H., Chalasani, S. H., Butcher, R. A., Clardy, J., et al. (2009). A hub-and-spoke circuit drives pheromone attraction and social behaviour in C. elegans. Nature 458, 1171-1175. doi: 10.1038/nature07886

Marder, E. (2009). Electrical synapses: rectification demystified. Curr. Biol. 19, R34R35. doi: 10.1016/j.cub.2008.11.008

Margiotta, J. F., and Walcott, B. (1983). Conductance and dye permeability of a rectifying electrical synapse. Nature 305, 52-55. doi: 10.1038/305052a0

Marks, W. D., and Skerrett, I. M. (2014). Role of the amino terminus in voltage gating and junctional rectification of Shaking B Innexins. J. Neurophysiol. 111, 1383-1395. doi: 10.1152/jn.00385.2013

Márquez-Rosado, L., Solan, J. L., Dunn, C. A., Norris, R. P., and Lampe, P. D. (2012). Connexin43 phosphorylation in brain, cardiac, endothelial and epithelial tissues. Biochim. Biophys. Acta 1818, 1985-1992. doi: 10.1016/j.bbamem. 2011.07.028 
Matsuda, H., Kurata, Y., Oka, C., Matsuoka, S., and Noma, A. (2010). Magnesium gating of cardiac gap junction channels. Prog. Biophys. Mol. Biol. 103, 102-110. doi: 10.1016/j.pbiomolbio.2010.05.009

Mauro, A. (1962). Space charge regions in fixed charge membranes and the associated property of capacitance. Biophys. J. 2, 179-198. doi: 10.1016/s00063495(62)86848-9

Muller, K. J., and Scott, S. A. (1981). Transmission at a "direct" electrical connexion mediated by an interneurone in the leech. J. Physiol. 311, 565-583.

Musa, H., Fenn, E., Crye, M., Gemel, J., Beyer, E. C., and Veenstra, R. D. (2004). Amino terminal glutamate residues confer spermine sensitivity and affect voltage gating and channel conductance of rat connexin40 gap junctions. J. Physiol. 557, 863-878. doi: 10.1113/jphysiol.2003.059386

Noma, A., and Tsuboi, N. (1987). Dependence of junctional conductance on proton, calcium and magnesium ions in cardiac paired cells of guinea-pig. $J$. Physiol. 382, 193-211.

Oh, S., Rubin, J. B., Bennett, M. V., Verselis, V. K., and Bargiello, T. A. (1999). Molecular determinants of electrical rectification of single channel conductance in gap junctions formed by connexins 26 and 32. J. Gen. Physiol. 114, 339-364. doi: 10.1085/jgp.114.3.339

Palacios-Prado, N., and Bukauskas, F. F. (2009). Heterotypic gap junction channels as voltage-sensitive valves for intercellular signaling. Proc. Natl. Acad. Sci. U S A 106, 14855-14860. doi: 10.1073/pnas.0901923106

Palacios-Prado, N., and Bukauskas, F. F. (2012). Modulation of metabolic communication through gap junction channels by transjunctional voltage; synergistic and antagonistic effects of gating and ionophoresis. Biochim. Biophys. Acta 1818, 1884-1894. doi: 10.1016/j.bbamem.2011.09.001

Palacios-Prado, N., Chapuis, S., Panjkovich, A., Fregeac, J., Nagy, J. I., and Bukauskas, F. F. (2014). Molecular determinants of magnesium-dependent synaptic plasticity at electrical synapses formed by connexin36. Nat. Commun. 5:4667. doi: 10.1038/ncomms5667

Palacios-Prado, N., Hoge, G., Marandykina, A., Rimkute, L., Chapuis, S., Paulauskas, N., et al. (2013). Intracellular magnesium-dependent modulation of gap junction channels formed by neuronal connexin36. J. Neurosci. 33, 47414753. doi: 10.1523/JNEUROSCI.2825-12.2013

Panchin, Y., Kelmanson, I., Matz, M., Lukyanov, K., Usman, N., and Lukyanov, S. (2000). A ubiquitous family of putative gap junction molecules. Curr. Biol. 10, R473-R474. doi: 10.1016/s0960-9822(00)00576-5

Pereda, A. E., Bell, T. D., and Faber, D. S. (1995). Retrograde synaptic communication via gap junctions coupling auditory afferents to the Mauthner cell. $J$. Neurosci. 15, 5943-5955.

Pereda, A. E., Curti, S., Hoge, G., Cachope, R., Flores, C. E., and Rash, J. E. (2013). Gap junction-mediated electrical transmission: regulatory mechanisms and plasticity. Biochim. Biophys. Acta 1828, 134-146. doi: 10.1016/j.bbamem. 2012.05.026

Pereda, A. E., Rash, J. E., Nagy, J. I., and Bennett, M. V. L. (2004). Dynamics of electrical transmission at club endings on the Mauthner cells. Brain Res. Brain Res. Rev. 47, 227-244. doi: 10.1016/j.brainresrev.2004.06.010

Perisse, E., Burke, C., Huetteroth, W., and Waddell, S. (2013). Shocking revelations and saccharin sweetness in the study of Drosophila olfactory memory. Curr. Biol. 23, R752-R763. doi: 10.1016/j.cub.2013.07.060

Phelan, P. (2005). Innexins: members of an evolutionarily conserved family of gap-junction proteins. Biochim. Biophys. Acta 1711, 225-245. doi: 10.1016/j. bbamem.2004.10.004

Phelan, P., Goulding, L. A., Tam, J. L., Allen, M. J., Dawber, R. J., Davies, J. A., et al. (2008). Molecular mechanism of rectification at identified electrical synapses in the Drosophila giant fiber system. Curr. Biol. 18, 1955-1960. doi: 10.1016/j.cub. 2008.10.067

Phelan, P., Nakagawa, M., Wilkin, M. B., Moffat, K. G., O’Kane, C. J., Davies, J. A., et al. (1996). Mutations in shaking-B prevent electrical synapse formation in the Drosophila giant fiber system. J. Neurosci. 16, 1101-1113.

Phelan, P., and Starich, T. A. (2001). Innexins get into the gap. Bioessays 23, 388396. doi: 10.1002/bies.1057

Phelan, P., Stebbings, L. A., Baines, R. A., Bacon, J. P., Davies, J. A., and Ford, C. (1998). Drosophila Shaking-B protein forms gap junctions in paired Xenopus oocytes. Nature 391, 181-184. doi: 10.1038/34426

Pitman, J. L., Huetteroth, W., Burke, C. J., Krashes, M. J., Lai, S. L., Lee, T., et al. (2011). A pair of inhibitory neurons are required to sustain labile memory in the Drosophila mushroom body. Curr. Biol. 21, 855-861. doi: 10.1016/j.cub.2011. 03.069
Rabinowitch, I., Chatzigeorgiou, M., and Schafer, W. R. (2013). A gap junction circuit enhances processing of coincident mechanosensory inputs. Curr. Biol. 23, 963-967. doi: 10.1016/j.cub.2013.04.030

Rackauskas, M., Kreuzberg, M. M., Pranevicius, M., Willecke, K., Verselis, V. K., and Bukauskas, F. F. (2007). Gating properties of heterotypic gap junction channels formed of connexins 40, 43 and 45. Biophys. J. 92, 1952-1965. doi: 10. 1529/biophysj.106.099358

Ramón, F., and Rivera, A. (1986). Gap junction channel modulation-a physiological viewpoint. Prog. Biophys. Mol. Biol. 48, 127-153. doi: 10.1016/00796107(86)90010-6

Rash, J., Curti, S., Vanderpool, K., Kamasawa, N., Nannapaneni, S., Palacios-Prado, N., et al. (2013). Molecular and functional asymmetry at a vertebrate electrical synapse. Neuron 79, 957-969. doi: 10.1016/j.neuron.2013.06.037

Rela, L., and Szczupak, L. (2003). Coactivation of motoneurons regulated by a network combining electrical and chemical synapses. J. Neurosci. 23, 682-692.

Rela, L., and Szczupak, L. (2007). In situ characterization of a rectifying electrical junction. J. Neurophysiol. 97, 1405-1412. doi: 10.1152/jn.00973.2006

Ringham, G. L. (1975). Localization and electrical characteristics of a giant synapse in the spinal cord of the lamprey. J. Physiol. 251, 395-407.

Roberts, A., Krasne, F. B., Hagiwara, G., Wine, J. J., and Kramer, A. P. (1982). Segmental giant: evidence for a driver neuron interposed between command and motor neurons in the crayfish escape system. J. Neurophysiol. 47, 761-781.

Sahu, G., Sukumaran, S., and Bera, A. K. (2014). Pannexins form gap junctions with electrophysiological and pharmacological properties distinct from connexins. Sci. Rep. 4:4955. doi: 10.1038/srep04955

Samuels, S. E., Lipitz, J. B., Dahl, G., and Muller, K. J. (2010). Neuroglial ATP release through innexin channels controls microglial cell movement to a nerve injury. J. Gen. Physiol. 136, 425-442. doi: 10.1085/jgp.201010476

Scemes, E., Suadicani, S. O., Dahl, G., and Spray, D. C. (2007). Connexin and pannexin mediated cell-cell communication. Neuron Glia Biol. 3, 199-208. doi: $10.1017 /$ S1740925X08000069

Shang, Y., Claridge-Chang, A., Sjulson, L., Pypaert, M., and Miesenböck, G. (2007). Excitatory local circuits and their implications for olfactory processing in the fly antennal lobe. Cell 128, 601-612. doi: 10.1016/j.cell.2006.12.034

Shindo, Y., Fujimoto, A., Hotta, K., Suzuki, K., and Oka, K. (2010). Glutamateinduced calcium increase mediates magnesium release from mitochondria in rat hippocampal neurons. J. Neurosci. Res. 88, 3125-3132. doi: 10.1002/jnr.22467

Simonsen, K. T., Moerman, D. G., and Naus, C. C. (2014). Gap junctions in C. elegans. Front. Physiol. 5:40. doi: 10.3389/fphys.2014.00040

Smith, T. G., Baumann, F., and Fuortes, M. G. (1965). Electrical connections between visual cells in the ommatidium of limulus. Science 147, 1446-1448. doi: $10.1126 /$ science.147.3664.1446

Söhl, G., Güldenagel, M., Traub, O., and Willecke, K. (2000). Connexin expression in the retina. Brain Res. Brain Res. Rev. 32, 138-145. doi: 10.1016/s01650173(99)00074-0

Söhl, G., and Willecke, K. (2004). Gap junctions and the connexin protein family. Cardiovasc. Res. 62, 228-232. doi: 10.1016/j.cardiores.2003.11.013

Starich, T. A., Herman, R. K., and Shaw, J. E. (1993). Molecular and genetic analysis of unc-7, a Caenorhabditis elegans gene required for coordinated locomotion. Genetics 133, 527-541.

Starich, T. A., Lee, R. Y., Panzarella, C., Avery, L., and Shaw, J. E. (1996). eat-5 and unc-7 represent a multigene family in Caenorhabditis elegans involved in cell-cell coupling. J. Cell Biol. 134, 537-548. doi: 10.1083/jcb.134.2.537

Starich, T. A., Xu, J., Skerrett, I. M., Nicholson, B. J., and Shaw, J. E. (2009). Interactions between innexins UNC-7 and UNC-9 mediate electrical synapse specificity in the Caenorhabditis elegans locomotory nervous system. Neural Dev. 4:16. doi: 10.1186/1749-8104-4-16

Stebbings, L. A., Todman, M. G., Phelan, P., Bacon, J. P., and Davies, J. A. (2000). Two Drosophila innexins are expressed in overlapping domains and cooperate to form gap-junction channels. Mol. Biol. Cell 11, 2459-2470. doi: 10.1091/mbc. 11.7.2459

Suchyna, T. M., Nitsche, J. M., Chilton, M., Harris, A. L., Veenstra, R. D., and Nicholson, B. J. (1999). Different ionic selectivities for connexins 26 and 32 produce rectifying gap junction channels. Biophys. J. 77, 2968-2987. doi: 10. 1016/s0006-3495(99)77129-8

Sun, Y.-A., and Wyman, R. J. (1996). Passover eliminates gap junctional communication between neurons of the giant fiber system in Drosophila. J. Neurobiol. 30, 340-348. doi: 10.1002/(sici)1097-4695(199607)30:3<340::aid-neu3>3. 3.co; $2-\mathrm{b}$ 
Swenson, K. I., Jordan, J. R., Beyer, E. C., and Paul, D. L. (1989). Formation of gap junctions by expression of connexins in Xenopus oocyte pairs. Cell 57, 145-155. doi: 10.1016/0092-8674(89)90180-3

Tanouye, M. A., and Wyman, R. J. (1980). Motor outputs of giant nerve fiber in Drosophila. J. Neurophysiol. 44, 405-421.

Teubner, B., Degen, J., Sohl, G., Guldenagel, M., Bukauskas, F. F., Trexler, E. B., et al. (2000). Functional expression of the murine connexin 36 gene coding for a neuron-specific gap junctional protein. J. Membr. Biol. 176, 249-262. doi: 10. 1007/s002320001094

Thomas, J. B., and Wyman, R. J. (1984). Mutations altering synaptic connectivity between identified neurons in Drosophila. J. Neurosci. 4, 530-538.

Tomer, R., Denes, A. S., Tessmar-Raible, K., and Arendt, D. (2010). Profiling by image registration reveals common origin of annelid mushroom bodies and vertebrate pallium. Cell 142, 800-809. doi: 10.1016/j.cell.2010. 07.043

Trenholm, S., McLaughlin, A. J., Schwab, D. J., and Awatramani, G. B. (2013). Dynamic tuning of electrical and chemical synaptic transmission in a network of motion coding retinal neurons. J. Neurosci. 33, 14927-14938. doi: 10. 1523/jneurosci.0808-13.2013

Tsien, J. Z. (2000). Linking Hebb's coincidence-detection to memory formation. Curr. Opin. Neurobiol. 10, 266-273. doi: 10.1016/S0959-4388(00)00070-2

Vaney, D. I., and Weiler, R. (2000). Gap junctions in the eye: evidence for heteromeric, heterotypic and mixed-homotypic interactions. Brain Res. Brain Res. Rev. 32, 115-120. doi: 10.1016/s0165-0173(99)00070-3

Venance, L., Glowinski, J., and Giaume, C. (2004). Electrical and chemical transmission between striatal GABAergic output neurones in rat brain slices. J. Physiol. 559, 215-230. doi: 10.1113/jphysiol.2004.065672

Verselis, V. K., Ginter, C. S., and Bargiello, T. A. (1994). Opposite voltage gating polarities of two closely related connexins. Nature 368, 348-351. doi: 10 1038/368348a0

Veruki, M. L., and Hartveit, E. (2002). AII (Rod) amacrine cells form a network of electrically coupled interneurons in the mammalian retina. Neuron 33, 935-946. doi: 10.1016/s0896-6273(02)00609-8

Volff, J.-N. (2005). Genome evolution and biodiversity in teleost fish. Heredity (Edinb). 94, 280-294. doi: 10.1038/sj.hdy.6800635

Watanabe, A. (1958). The interaction of electrical activity among neurons of lobster cardiac ganglion. Jpn. J. Physiol. 8, 305-318. doi: 10.2170/jjphysiol.8.305

Weidmann, S. (1952). The electrical constants of Purkinje fibres. J. Physiol. 118, $348-360$.
Werner, R., Levine, E., Rabadan Diehl, C., and Dahl, G. (1989). Formation of hybrid cell-cell channels. Proc. Natl. Acad. Sci. U S A 86, 5380-5384. doi: 10.1073/pnas. 86.14.5380

White, R. L., Doeller, J. E., Verselis, V. K., and Wittenberg, B. A. (1990). Gap junctional conductance between pairs of ventricular myocytes is modulated synergistically by H+ and Ca++. J. Gen. Physiol. 95, 1061-1075. doi: 10.1085/jgp. 95.6.1061

Wilson, R. I. (2013). Early olfactory processing in Drosophila: mechanisms and principles. Annu. Rev. Neurosci. 36, 217-241. doi: 10.1146/annurev-neuro062111-150533

Wu, C.-L., Shih, M.-F. M., Lai, J. S.-Y., Yang, H.-T., Turner, G. C., Chen, L., et al. (2011). Heterotypic gap junctions between two neurons in the Drosophila brain are critical for memory. Curr. Biol. 21, 848-854. doi: 10.1016/j.cub.2011.02.041

Yaksi, E., and Wilson, R. I. (2010). Electrical coupling between olfactory glomeruli. Neuron 67, 1034-1047. doi: 10.1016/j.neuron.2010.08.041

Yamanaka, R., Shindo, Y., Hotta, K., Suzuki, K., and Oka, K. (2013). $\mathrm{NO} / \mathrm{cGMP} / \mathrm{PKG}$ signaling pathway induces magnesium release mediated by mitoKATP channel opening in rat hippocampal neurons. FEBS Lett. 587, $2643-$ 2648. doi: 10.1016/j.febslet.2013.06.049

Zhang, Z., Curtin, K. D., Sun, Y.-A., and Wyman, R. J. (1999). Nested transcripts of gap junction gene have distinct expression patterns. J. Neurobiol. 40, 288-301. doi: 10.1002/(sici)1097-4695(19990905)40:3<288::aid-neu2>3.0.co;2-o

Conflict of Interest Statement: The authors declare that the research was conducted in the absence of any commercial or financial relationships that could be construed as a potential conflict of interest.

Received: 09 July 2014; accepted: 26 September 2014; published online: 15 October 2014.

Citation: Palacios-Prado N, Huetteroth $W$ and Pereda AE (2014) Hemichannel composition and electrical synaptic transmission: molecular diversity and its implications for electrical rectification. Front. Cell. Neurosci. 8:324. doi: 10.3389/fncel.2014.00324 This article was submitted to the journal Frontiers in Cellular Neuroscience.

Copyright (c) 2014 Palacios-Prado, Huetteroth and Pereda. This is an open-access article distributed under the terms of the Creative Commons Attribution License (CC $B Y)$. The use, distribution and reproduction in other forums is permitted, provided the original author(s) or licensor are credited and that the original publication in this journal is cited, in accordance with accepted academic practice. No use, distribution or reproduction is permitted which does not comply with these terms. 